\title{
Expression of aquaporin 1 and 4 in rats with acute hypoxic lung injury and its significance
}

\author{
Y. Wu1, C.Y. Pan' ${ }^{2}$, C.Z. Guo', Z.J. Dong' ${ }^{1}$, Q. Wu1 ${ }^{1}$, H.M. Dong ${ }^{3}$ and W. Zhang ${ }^{1}$ \\ 1Department of Pathology, Medical College of QingHai University, \\ XiNing, QingHai Province, China \\ ${ }^{2}$ Department of Pathology, Nanyang Medical College, NanYang, \\ HeNan Province, China \\ ${ }^{3}$ Chinese People's Liberation Army Fourth Army Hospital, QingHai Province, China \\ Corresponding author: W. Zhang \\ E-mail: qhzhangwei_|@163.com
}

Genet. Mol. Res. 14 (4): 12756-12764 (2015)

Received April 22, 2015

Accepted July 23, 2015

Published October 19, 2015

DOI http://dx.doi.org/10.4238/2015.October.19.19

ABSTRACT. Aquaporin (AQP)-1 and AQP-4 expression in lung tissues of $S D$ rats during high altitude hypoxic lung injury, and the relationship between AQP-1 and AQP-4 expression, and acute hypoxic lung injury was analyzed. Thirty six healthy SD rats were divided into hypoxia $1 d, 2 d, 3 d, 5 d$, and $7 \mathrm{~d}$ groups and control group $(\mathrm{N}=6)$. Pathological changes in lung tissue were observed by hematoxylin and eosin staining; lung injury was scored, and ultrastructural changes in lung tissue were observed by transmission electron microscopy. Changes in moisture content in lung tissues were determined by analyzing the wet/dry weight ratio (W/D). Localization of AQP-1 and AQP-4 was determined by immunohistochemistry. AQP-1 and AQP-4 expression were detected by western blot. Lung W/D was lower in hypoxia groups than in control group, and the highest in $3 d$ group $(P<0.05)$. Light microscopy revealed a thickening alveolar wall and outstretched and congestive alveolar wall in hypoxia group; electron microscopy revealed 
the presence of abnormal alveolar type II epithelial cells, cavitation in cytoplasm, microvillus-like protrusions, and a reduced lamellar body. AQP1 and AQP-4 were mainly distributed in the capillaries and lymphatic and alveolar epithelial cells and airway epithelial cells, respectively. AQP-1 protein expression was decreased (western blot) in hypoxia $1 \mathrm{~d}$ group (the lowest in 3d group; $P<0.05$ ); there were no significant changes about AQP4 expression. Therefore, AQP-1 may be involved in abnormal transport of liquid $A L I$ and pathogenesis of lung edema. AQP-4 may not be involved in the formation of ALI lung edema.

Key words: High altitude hypoxia; Lung injury; AQP-1; AQP-4

\section{INTRODUCTION}

Acute lung injury $(A L I)$ is a clinical syndrome characterized by highly permeable lung edema; so far, its pathogenesis has not been fully elucidated. Alveolar water transport was believed to be associated with the active transport of sodium, because of the lack of awareness of the function of aquaporins (AQPs) in lung tissues. AQPs comprise a group of cell membrane transport proteins that are involved in the transport of liquid under physiological conditions, as well as the pathological state of fluid transport. At present, studies on the function of AQPs in $A L I$ are mainly focused on AQP-1 and AQP-5, with research on AQP-4 lacking. Research into whether AQP-1 and AQP-4 expression in the lung tissue is increased or decreased following ALI remains controversial. The changes in expression of AQP-1 and AQP-4 in lung tissues after acute hypoxia ALI have not been previously reported. In this study, we have investigated the changes in expression of AQP-1 and AQP-4 in lung tissues using a low-pressure oxygen cabin to simulate the ALI model of rats, and to elucidate its role in lung edema in ALI rats during the early hypoxic stage.

\section{MATERIAL AND METHODS}

\section{Animals and reagents}

Forty healthy adult SD rats (specific pathogen-free level) were purchased from the animal experiment center of Xi'an Jiao Tong University; the weight of these rats ranged from 150 to 250 g. The AQP-1, AQP-4, and GAPDH antibodies, goat-anti rabbit IgG, and DAB Color kit were purchased from WuHan Boster Biological Technology Co., Ltd., WuHan City, China.

\section{Animal models and grouping}

Thirty-six SD rats were purchased from Xi'an (altitude $400 \mathrm{~m}$ ), their weights ranging from 210 to $230 \mathrm{~g}$; these were randomly divided into 6 groups $(\mathrm{N}=6)$. Rats in the control group were tested in the plains (altitude $400 \mathrm{~m}$ ), the other 5 groups were quickly transported to the Qumalai county of Qinghai Province, with an altitude of $4500 \mathrm{~m}$, within $10 \mathrm{~h}$; the experiments were conducted in high altitude at 1, 2, 3, 5, and 7 days post-transport. The related indices were tested at the same time. 


\section{Determination of lung wet/dry ratio (W/D)}

The middle left lung tissue of rats of each group was baked in an oven at $80^{\circ} \mathrm{C}$ for $24 \mathrm{~h}$, until a constant weight was reached. The weights of the lung tissues were recorded before and after baking, and the W/D ratio was calculated.

\section{Morphology of lung tissue}

The left lung apex of rats was obtained and fixed with $4 \%$ paraformaldehyde; the tissues were cut into $4-\mu \mathrm{m}$ thick slices and observed by hematoxylin and eosin (H\&E staining after dehydration and embedding in paraffin. The morphology was scored using the Smith pathological lung injury score (Smith et al., 1997). The tissues were scored according to the four aspects of lung edema: alveolar and lung interstitial inflammation, alveolar and interstitial hemorrhage, and lung atelectasis. The scores ranged from 0 to 4 points: 0 indicated a normal lung, 1, 2, 3, and 4 indicated mild, moderate, severe, and extremely severe inflammation.

\section{Ultrastructure of lung tissue}

Tissues were obtained from the middle lobe of the right lungs of rats, fixed with glutaraldehyde, immersed in $0.1 \mathrm{M}$ phosphate buffer solution, and again fixed with $1 \%$ osmium tetroxide. The samples were then dehydrated using an ethanol gradient; subsequently, the ethanol was replaced with propylene oxide, and the samples were embedded using epoxy resin 618 . The ultrathin sections were stained with uranyl acetate lead citrate, and the ultrastructural changes in the lung tissues were observed under a transmission electron microscope.

\section{Distributions of AQP-1 and AQP-4 in lung tissue observed by immunohistochemistry}

The strept avidin-biotin complex (SABC) method was used to subject the slice to regular dewaxing and hydration; the endogenous enzymes were removed with $3 \%$ hydrogen peroxide and repaired with high-pressure antigen. The remaining steps were conducted according to the protocols provided by the manufacturers of the kit [SABC-POD(F) rabbit IgG kit, SA1028, Boster]. PBS was used as the negative control to instead of the primary antibody. AQP-1 and AQP-4 antibodies were diluted to 1:200. Intracellular brown coloring showed positive expression of AQP-1 and AQP-4 in the cells.

\section{Expression of $A Q P-1$ and $A Q P-4$ in lung tissues detected via western blot}

Forty milligram of the frozen tissue was taken and $200 \mu \mathrm{L}$ protein lysate was added to every $10 \mathrm{mg}$ of the tissue; a tissue homogenate was obtained using electric grinder at $4^{\circ} \mathrm{C}$ on ice. The homogenate was centrifuged at $12,000 \mathrm{~g}$ for $20 \mathrm{~min}$. The supernatant was packed into a 1.5$\mathrm{mL}$ centrifuge tube and preserved at $-80^{\circ} \mathrm{C}$. In strict accordance with the manufacturer instructions, the samples obtained were electrophoresed, transferred onto a film, and incubated with the primary antibody, secondary antibody, and the coloring agent in sequence. The color film was scanned and gray analysis performed using the ImageJ software; subsequently, the relative gray value was calculated. 


\section{Statistical analysis}

All data are reported as means \pm standard deviation; each group was subjected to a single factor analysis of variance using the SPSS v.17.0 statistical software (IBM, Armonk, NY, USA).

\section{RESULTS}

\section{General situation}

The rats in the hypoxic one-day (1d) group showed a good reaction and a ruddy complexion; the rats in hypoxic two-day (2d) group showed better reactions, a reduced diet intake and mental fatigue. The rats in the hypoxic three-day (3d) group were dispirited, appeared anorexic, and exhibited diarrhea, shortness of breath, nose and lip cyanosis, and irritability; rats in the hypoxia five-day (5d) group showed mild cyanosis and mental vibration; while the rats in the hypoxia seven-day (7d) group showed weight loss and anorexia, but no cyanosis.

\section{Ratio of W/D in lung tissue}

The W/D of lung tissues in the experiment group differed significantly from that of the control group; in addition, the W/D of the hypoxia 3d group also differed significantly from those of the other experimental groups $(P<0.05)$.

\section{H\&E staining of lung tissue}

The nucleus was stained blue and cytoplasm stained pink under a light microscope. The lung tissues of rats in the normal control group showed a clear structure, with no lung interstitial edema and hyperemia in the capillary. The hypoxia $1 \mathrm{~d}$ group exhibited alveolar cavity enlargement, alveolar wall thickening, interstitial lung capillary dilation, and congestion, and inflammatory cell infiltration. The rats in the hypoxia $2 d$ group showed aggravated lung injury, while the hypoxia $3 d$ group exhibited lung interstitial edema, continued alveolar wall thickening, reduced alveolar cavity, interstitial lung capillary dilation, reduced number of red blood cells in the alveoli, and increased infiltration of inflammatory cells. The lung injury of rats increased with the increase in time of hypoxia; the thickening of the alveolar wall was mitigated in rats of the hypoxia $5 \mathrm{~d}$ group, and rats in the hypoxia $7 d$ group exhibited infiltration of a few neutrophils in the alveoli and interstitial lung, with a continuous reduction in the thickening of the alveolar wall (Figure $1 \mathrm{~A}-\mathrm{F}$ ). Determination of the pathological score of each group indicated that the scores of the hypoxia groups were higher than those of the control group $(P<0.05)$, with the hypoxia $3 d$ group displaying the highest score $(P<0.05$; Table 1$)$.

\section{Ultrastructure of lung tissue}

The TEM ultrastructure of the lung tissues of rats from the hypoxia $1 \mathrm{~d}$ group showed abnormalities in the alveolar type II epithelial cells, decreased microvilli, vacuolar cytoplasm, and decreased lamellar bodies. The hypoxia $2 \mathrm{~d}$ group displayed swollen mitochondria in the cytoplasm of alveolar type II cells and slight hyperplasia in the alveolar mediastinum. We observed a reduction in the alveolar type II cells and partial lamellar bodies in the cytoplasm in the hypoxia $3 \mathrm{~d}$ group. The ultrastructure of lung tissue in the hypoxia $5 \mathrm{~d}$ group showed mild abnormalities in the alveolar type Il cells, reduced membrane microvilli, dilation of cytoplasmic endoplasmic reticulum, and a small 
quantity of lamellar structures; abnormalities in the alveolar type II cells and plasma membrane protrusions were considerably reduced in the hypoxia $7 \mathrm{~d}$ group. However, the cytoplasm displayed a slightly more plate layer structure (Figure $2 \mathrm{~A}-\mathrm{F})$.

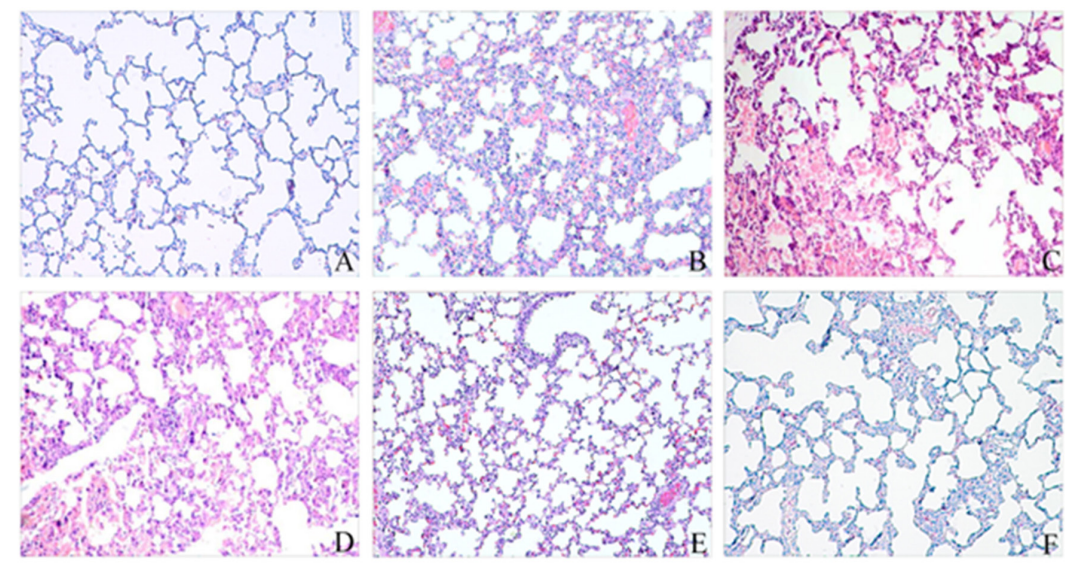

Figure 1. H\&E staining of lung tissues in each group (100X). A. Normal lung tissues of the control group. B. Hypoxia 1d group. C. Hypoxia 2d group. D. Hypoxia 3d group. E. Hypoxia 5d group. F. Hypoxia 7d group.

Table 1. Indices of rats with hypoxic lung injury.

\begin{tabular}{llcccc}
\hline Group & N & W/D & Smith score & AQP-1 (Gray ratio with GAPDH) & AQP-4 (Gray ratio with GAPDH) \\
\hline Control group & 6 & $5.28 \pm 0.60$ & $0.34 \pm 0.10$ & $1.498 \pm 0.026$ & $1.032 \pm 0.016$ \\
1 day & 6 & $7.69 \pm 0.33^{*}$ & $2.67 \pm 0.52^{*}$ & $1.106 \pm 0.027^{\star}$ & $1.025 \pm 0.043$ \\
2 day & 6 & $9.11 \pm 0.82^{*}$ & $6.81 \pm 0.43^{*}$ & $0.665 \pm 0.056^{*}$ & $1.028 \pm 0.036$ \\
3 day & 6 & $9.86 \pm 1.38^{* \Delta}$ & $7.50 \pm 1.52^{\star_{\Delta}}$ & $0.434 \pm 0.038^{*}$ & $1.037 \pm 0.012$ \\
5 day & 6 & $8.25 \pm 1.01^{*}$ & $4.93 \pm 1.07^{*}$ & $0.602 \pm 0.074^{*}$ & $1.026 \pm 0.060$ \\
7 day & 6 & $7.12 \pm 0.21^{*}$ & $2.00 \pm 0.89^{*}$ & $1.305 \pm 0.015^{\star}$ & $1.019 \pm 0.027$ \\
\hline
\end{tabular}

${ }^{*}$ Compared to the normal control group, $\mathrm{P}<0.05 ;{ }^{\wedge}$ Comparison with each experimental group, $\mathrm{P}<0.05$.
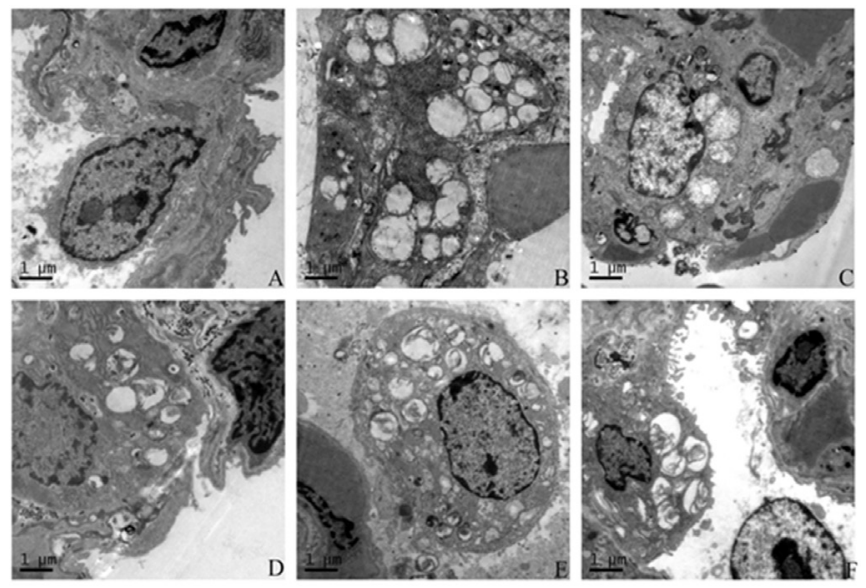

Figure 2. Ultrastructure of a lung tissue (10,000X). A. Normal lung tissue of the control group. B. Hypoxia 1d group. C. hypoxia 2 d group. D. Hypoxia 3d group. E. Hypoxia 5d group. F. Hypoxia $7 d$ group. 


\section{Changes in AQP1 and AQP4 expression in lung tissues observed by immunohistochemistry}

We observed a high level of expression of AQP-1 in the lung tissues of the control group, and an obvious yellow-to-brown distribution in the lung capillaries and alveolar endothelial cells. Compared to the control group, the simulation plateau hypoxia groups showed decreased pigmentation indicating the presence of the AQP-1 protein in the lung tissues. AQP-4 was mainly distributed in the airway epithelial cells; we observed no significant changes in the distribution and expression of AQP-4 during lung injury compared to the control group (Figures 3A-F and 4A-F).
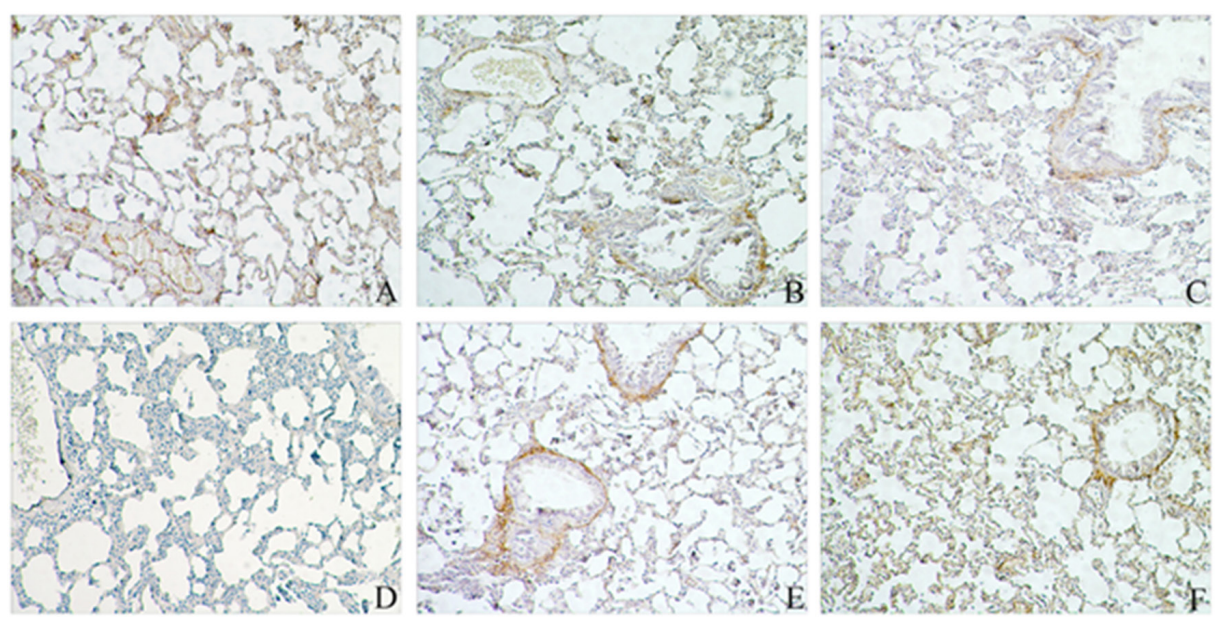

Figure 3. Expression of AQP-1 in the lung tissue of each hypoxia group (SABC 200X). A. Normal lung tissue of the control group. B. Hypoxia 1d group. C. Hypoxia 2d group. D. Hypoxia 3d group. E. hypoxia 5d group. F. Hypoxia 7d group.
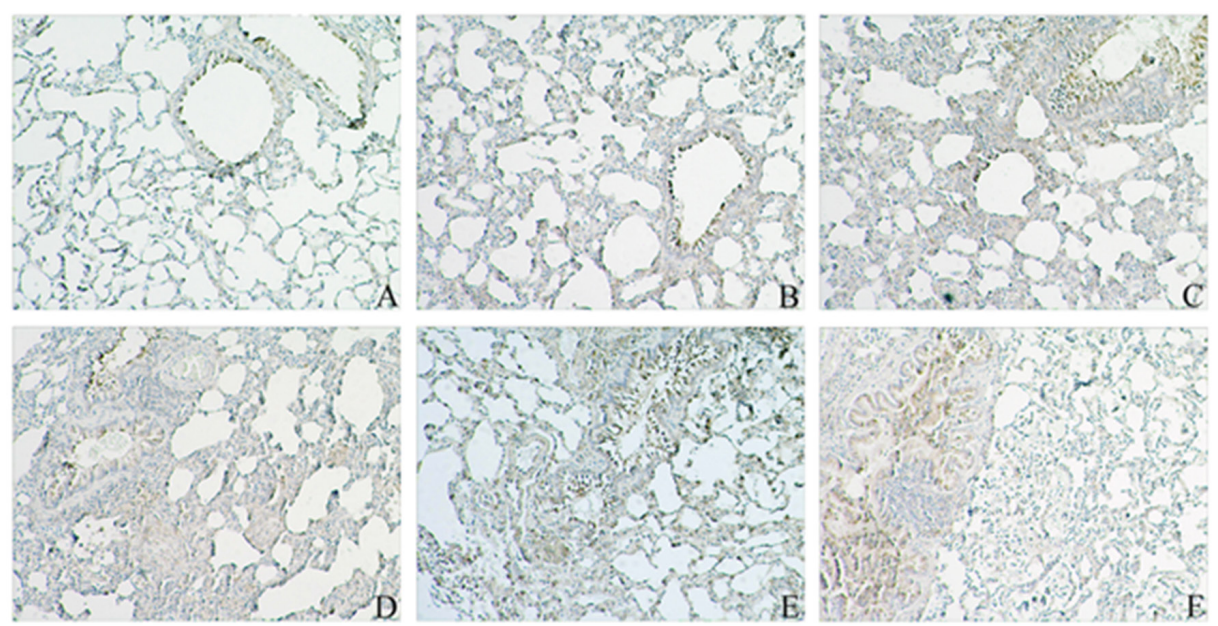

Figure 4. Expression of AQP-4 in the lung tissues of each hypoxia group (SABC 200X). A. Normal lung tissue of the control group. B. Hypoxia 1d group. C. Hypoxia 2d group. D. Hypoxia 3d group. E. hypoxia 5d group. F. Hypoxia 7d group. 


\section{Changes in AQP1 and AQP4 expression in the lung tissue of each group, analyzed by western blot}

The expression of AQP1 in the lung tissues of hypoxic rats was decreased compared to the normal control group. AQP-1 expression reached a peak value in the hypoxic $3 \mathrm{~d}$ group; this difference was statistically significant. We observed no significant changes in the expression of AQP4 in any of the rats $(P<0.05$; Table 1 , Figures 5 and 6$)$.

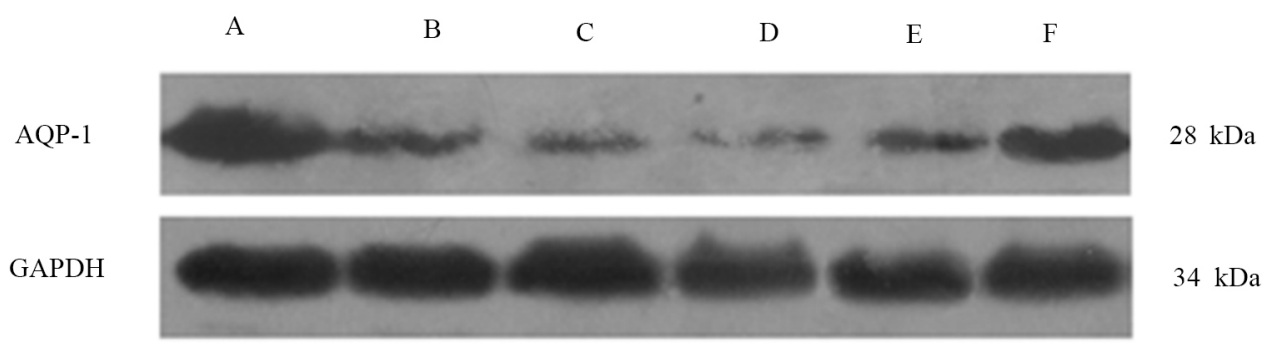

Figure 5. Western blot results of AQP-1 in rat lung tissues. A. Normal lung tissue of the control group. B. hypoxia 1d group. C. Hypoxia 2d group. D. Hypoxia 3d group. E. Hypoxia 5d group. F. Hypoxia 7d group.

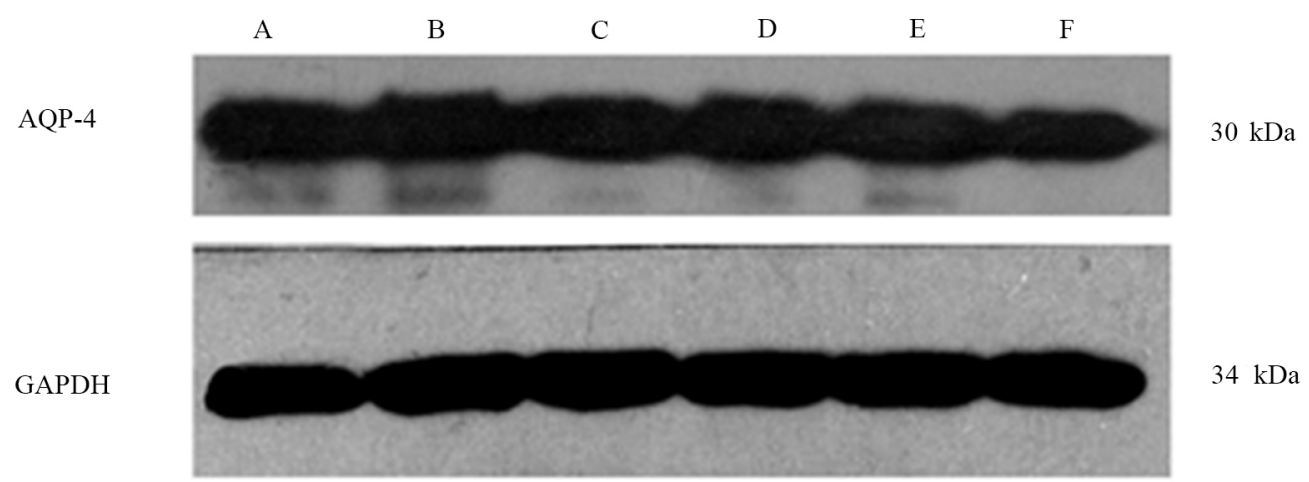

Figure 6. Western blot results of AQP-4 in rat lung tissues. A. Normal lung tissue of the control group. B. Hypoxia 1d group. C. Hypoxia 2d group. D. Hypoxia 3d group. E. Hypoxia 5d group. F. Hypoxia 7d group.

\section{DISCUSSION}

Hypoxia increases the vascular permeability of the lung and destroys the fluid balance in, and water barrier of lung tissues, which leads to severe ALI (lung edema). The pathological characteristics of lung edema include fluid accumulation in the alveolar and interstitial lung. Many research studies have shown that AQP is involved in the pathogenesis of lung edema (Singha et al., 2013). At present, 13 types of AQPs have been found in mammals (AQP 0-12), 6 of which are distributed in the lung tissues (AQP-1, -3, -4, -5, -8, and -9) (Zhu et al., 2008). Bai et al. (1999) also found a 10 -fold decrease in the water permeability of the alveolar capillary membrane barrier following an AQP-1 gene knockout. Meanwhile, the water permeability was decreased 14 to 16 times after AQP-1 and AQP-4 knockout in mice. This indicated that AQPs selectively 
allowed the passage of water molecules and mediated the transmembrane transport of free water molecules; therefore, these proteins are believed to closely influence the lung water balance and the occurrence of lung edema. However, the changes in AQP expression in the lung tissues as a result of ALI still need to be properly quantified. A majority of the studies showed a decrease in AQP expression (Towne et al., 2000); however, Lai et al. (2003) reported that inflammatory cytokines promote the expression of AQP-1 in a cell model. The expression of AQP-1 decreased significantly after subjecting them to 4-12 $\mathrm{h}$ of acute lung injury induced by acute pancreatitis. AQP-1 expression increased gradually after $12 \mathrm{~h}$ (Liu et al., 2014). Zhu et al. (2008), on the other hand, observed an increase in AQP-4 expression in rats with oleic acid-induced acute lung injury. Conversely, Li et al. (2008) reported that AQP-4 was not involved in the formation of ALI lung edema. These changes could possibly be attributed to the altered expression of AQP-1 and AQP4 during the early stages of high altitude hypoxia, resulting in abnormal transmembrane transport and accumulation of water in the lungs, which in turn damaged the lung tissue. At present, this has not been reported in literature. In this study, a high altitude hypoxia lung injury animal model was simulated in SD rats, and the AQP-1 and AQP-4 expression in lung tissues were analyzed over a long period of time in the context of radical plateau hypoxia; we also investigated the relationship between hypoxic ALI and AQP-1 and -4 .

The experimental results showed an increase in the lung W/D ratio in the hypoxic $1 \mathrm{~d}$ group; the hypoxic $3 d$ group showed the highest W/D, which decreased subsequently. This suggested a gradual increase in the lung fluid leakage during the acute phase of lung injury, and a high amount of exudate on day 3. Light microscopy revealed the thickening of the alveolar wall in experimental rats; in addition, the capillary was enlarged and filled with blood, and there was increased infiltration of red blood cells and macrophages in the alveoli and interstitial cells. Therefore, the pathological score of the lung tissue of rats in the experimental group was higher than that of rats in the control group $(P<0.05)$; the hypoxic 3d group showed the highest pathological score $(P<$ 0.05). Different degrees of abnormal alveolar type II epithelial cells were seen in the experimental groups under an electron microscope; in addition, we observed swelling of the mitochondria in the cytoplasm, a decrease in microvilli, cavitation, and reduction in/emptying of the plate layer. The injuries were most obvious in the hypoxia $3 d$ group, and the degree of the injury degree was mitigated in the $7 \mathrm{~d}$ group. This suggested that the hypoxic environment might have caused $A L I$ in rats. Immunohistochemistry revealed that AQP-1 was mainly expressed in the endothelial cells of capillary, around the bronchi and alveolar type II epithelial cells, while AQP-4 was mainly expressed in the airway epithelial cells. Results of the western blot analysis indicated a decrease in the expression of AQP-1 in hypoxia 1d rats, with the lowest level in 3d rats; AQP-1 expression increased gradually after 3 days $(P<0.05)$. AQP-1 is distributed in the peripheral lung vascular endothelium and bronchus, and is responsible for eliminating water from the bronchi and around the vessels. The levels of AQP-1 expression and the severity of lung edema are significantly related, suggesting an important association between AQP-1 and the formation of lung interstitial edema (resulting from dysfunction of the water clearance around the bronchi and vessels). We speculated that some inflammatory mediators might have damaged the endothelial cells during the early stage of hypoxic lung injury, or that alveolar type II epithelial cell apoptosis decreased the expression of AQP-1 in rat lung tissues, resulting in a decrease in the alveolar capillary water permeability and the scavenging capacity of lung tissues. This could in turn affect the absorption of edema fluid and aggravate the alveolar and interstitial edema, leading to the formation of lung edema. We observed no changes in the expression of AQP-4 in any of the group; this was attributed to the distribution 
of AQP-4 in the airway epithelial cells. The main function of AQP-4 is to remove moisture from the airway; however, as lung injuries are mainly caused by lesions in the alveolar wall, with minimal damage to the airway epithelial cells, the expression of AQP-4 remained chiefly unaltered.

This study showed that AQP-1 plays a regulatory role in the formation of lung interstitial edema and the transport and clearance of water during the different stages of acute hypoxic lung injury in a rat model. The AQP-4 expression showed no obvious changes, indicating that it may not participate in the development of lung edema after ALI. The role of AQPs in the occurrence and development of lung damage must be further researched, in order to develop methods to improve the content or activity of AQPs and increase the water clearance of lungs in patients to treat acute altitude-related hypoxic lung injury.

\section{Conflicts of interest}

The authors declare no conflict of interest.

\section{ACKNOWLEDGMENTS}

Research supported by the Foundation Item: The Qinghai University Youth Fund Project (\#2012.QYY-2), and grants provided by the National Natural Science Foundation of China (\#81160012) and the National Ministry of Health industry-specific (\#201002012).

\section{REFERENCES}

Bai C, Fukuda N, Song Y, Ma T, et al. (1999). Lung fluid transport in aquaporin-1 and aquaporin-4 knockout mice. J. Clin. Invest. 103: 555-561.

Lai KN, Leung JC, Metz CN, Lai FM, et al. (2003). Role of macrophage migration inhibitory factor in acute respiratory distress syndrome. J. Pathol. 199: 496-508.

Li B, Chen D and Wang GF (2008). The expression of aquaporin 1, 3, 4, 5 in lung tissue of mouse with endotoxin-induced acute lung injury. Ti Erh Chun i Ta Hsueh Hsueh Pao 29: 131-135.

Liu L, Du L, Chen Y, Qin S, et al. (2014). Down-regulation of aquaporin1 (AQP1) by peptidoglycan via p38 MAPK pathways in primary rat pleural mesothelial cells. Exp. Lung Res. 40: 145-153.

Singha O, Kengkoom K, Chaimongkolnukul K, Cherdyu S, et al. (2013). Pulmonary edema due to oral gavage in a toxicological study related to aquaporin-1, -4, and -5 expression. J. Toxicol. Pathol. 6: 283-291.

Smith KM, Mrozek JD, Simonton SC, Bing DR, et al. (1997). Prolonged partial liquid ventilation using conventional and highfrequency ventilatory techniques: gas exchange and lung pathology in an animal model of respiratory distress syndrome. Crit. Care Med. 25: 1888-1897.

Towne JE, Harrod KS, Krane CM and Menon AG (2000). Decreased expression of aquaporin (AQP)1 and AQP5 in mouse lung after acute viral infection. Am. J. Respir. Cell Mol. Biol. 22: 34-44.

Zhu LH, Li TP and He L (2008). The experimental study of aquaporin 4 in lung water metabolism in the early stage of acute lung injury induced by oleic acid. Nan Fang Yi Ke Da Xue Xue Bao 28: 707-711. 